\title{
New Sufficient Conditions for Hamiltonian and Traceable Graphs
}

\section{Rao Li}

Dept. of mathematical sciences, University of South Carolina Aiken, Aiken, SC 29801

raol@usca.edu

Abstract: In this note, we present new sufficient conditions for Hamiltonian and traceable graphs.

Keywords: Hamiltonian graph, traceable graph

\section{INTRODUCTION}

We consider only finite undirected graphs without loops or multiple edges. Notation and terminology not defined here follow those in [1]. For a graph $G=(V, E)$, we use $n$ and e to denote its order $|V|$ and size $|E|$, respectively. The connectivity of the graph $\mathrm{G}$ is denoted by $\mathrm{k}(\mathrm{G})$. For disjoint subsets $\mathrm{S}$, $\mathrm{T}$ of the vertex set $\mathrm{V}(\mathrm{G})$ of a graph $\mathrm{G}$, let $\mathrm{E}(\mathrm{S}, \mathrm{T})$ be the set of the edges in $\mathrm{G}$ that join a vertex in $\mathrm{S}$ and a vertex in T. We use GvH to denote the join of two disjoint graphs $\mathrm{G}$ and $\mathrm{H}$. The graph consists of $\mathrm{p}$ isolated vertices is denoted by Ep. A cycle $\mathrm{C}$ in a graph $\mathrm{G}$ is called a Hamiltonian cycle of $\mathrm{G}$ if $\mathrm{C}$ contains all the vertices of $\mathrm{G}$. A graph $\mathrm{G}$ is called Hamiltonian if $\mathrm{G}$ has a Hamiltonian cycle. A path $P$ in a graph $G$ is called a Hamiltonian path of $G$ if $P$ contains all the vertices of G. A graph $\mathrm{G}$ is called traceable if $\mathrm{G}$ has a Hamiltonian path. In this note, we present new sufficient conditions for Hamiltonian and traceable graphs. The main results are as follows.

Theorem 1. Let $\mathrm{G}$ be a graph of order $\mathrm{n} \geq 3$, e edges, and connectivity $\mathrm{k} \geq 2$. If

$$
\mathrm{e} \geq(\mathrm{n}-\mathrm{k}-1)(\mathrm{n}+\mathrm{k}) / 2,
$$

then $\mathrm{G}$ is Hamiltonian or $\mathrm{K}_{\mathrm{k}} \mathrm{vE}_{\mathrm{k}+1}$, where $\mathrm{n}=2 \mathrm{k}+1$.

Theorem 2. Let $G$ be a graph of order $n \geq 2$, e edges, and connectivity $k \geq 1$. If

$$
\mathrm{e} \geq(\mathrm{n}-\mathrm{k}-2)(\mathrm{n}+\mathrm{k}+1) / 2,
$$

then $\mathrm{G}$ is traceable or $\mathrm{K}_{\mathrm{k}} \mathrm{vE} \mathrm{k}_{\mathrm{k}}$, where $\mathrm{n}=2 \mathrm{k}+2$.

Next, we will prove Theorem 1 and Theorem 2.

\section{Proofs}

Proof of Theorem 1. Let G be a graph satisfying the conditions in Theorem 1. If G has a Hamiltonian cycle, then the proof is finished. Now we assume that $G$ is not Hamiltonian. Choose a longest cycle $C$ in $G$ and give an orientation on C. Since G is not Hamiltonian, there exists a vertex $x_{0}$ in $V(G)-V(C)$. By Menger's theorem, we can find $s(s \geq k)$ pairwise disjoint (except for $x_{0}$ ) paths $P_{1}, P_{2}, \ldots, P_{s}$ between $x_{0}$ and $V(C)$. Let $u_{i}$ be the end vertex of $\mathrm{P}_{\mathrm{i}}$ on $\mathrm{C}$, where $1 \leq \mathrm{i} \leq \mathrm{s}$. We, without loss of generality, assume that the appearance of $\mathrm{u}_{1}, \mathrm{u}_{2}, \ldots, \mathrm{u}_{\mathrm{s}}$ on $\mathrm{C}$ agrees with the given orientation of $\mathrm{C}$. We $u$ se $\mathrm{u}_{\mathrm{i}}^{+}$to denote the successor of $\mathrm{u}_{\mathrm{i}}$ along the given orientation of $\mathrm{C}$, where $1 \leq \mathrm{i} \leq \mathrm{s}$. Then a standard proof in Hamiltonian graph theory yields that $\mathrm{S}:=\left\{\mathrm{x}_{0}, \mathrm{u}_{1}^{+}, \mathrm{u}_{2}^{+}, \ldots, \mathrm{u}_{\mathrm{k}}^{+}\right\}$is independent (otherwise $\mathrm{G}$ would have cycles which are longer than $\mathrm{C}$ ). Thus

$$
\begin{aligned}
& (\mathrm{n}-\mathrm{k}-1)(\mathrm{n}+\mathrm{k}) / 2 \leq \mathrm{e}=|\mathrm{E}(\mathrm{S}, \mathrm{V}-\mathrm{S})|+|\mathrm{E}(\mathrm{G}[\mathrm{V}-\mathrm{S}])| \\
& \leq|\mathrm{S}|(\mathrm{n}-|\mathrm{S}|)+(\mathrm{n}-|\mathrm{S}|)(\mathrm{n}-|\mathrm{S}|-1) / 2 \\
& =(\mathrm{n}-|\mathrm{S}|)(\mathrm{n}+|\mathrm{S}|-1) / 2 \leq(\mathrm{n}-\mathrm{k}-1)(\mathrm{n}+\mathrm{k}) / 2 .
\end{aligned}
$$

Therefore $|S|=k+1$, xy is in $E$ for any vertex $x$ in $S$ and for any vertex y in $V-S$, and G[V - S)] is complete. 
Let $\mathrm{H}$ be the component of $\mathrm{G}[\mathrm{V}-\mathrm{V}(\mathrm{C})]$ containing $\mathrm{x}_{0}$. Since $\mathrm{u}_{1}{ }^{+}$is adjacent to any vertex in $\mathrm{V}-\mathrm{S}, \mathrm{H}$ must consist of a singleton $\mathrm{x}_{0}$ (otherwise $\mathrm{G}$ would have a cycle which is longer than $C$ ). Since $\mathrm{x}_{0}$ is adjacent to any vertex in $\mathrm{V}-\mathrm{S}$, $\mathrm{H}$ must be the only component of $\mathrm{G}[\mathrm{V}-\mathrm{V}(\mathrm{C})]$ (otherwise $\mathrm{x}_{0}$ would be adjacent to a vertex in another component of $\mathrm{G}[\mathrm{V}-\mathrm{V}(\mathrm{C})]$, which is a contradiction). Again, since $\mathrm{x}_{0}$ is adjacent to any vertex in $\mathrm{V}-\mathrm{S}$, the segment from $\mathrm{u}_{\mathrm{i}}{ }^{+}$to $u_{i+1}$ along the given orientation of $C$, for each $i$ with $1 \leq i \leq s$ and $s+1$ is regarded as 1 , must consist of only $u_{i}{ }^{+}$ and $u_{i+1}$ (otherwise $G$ would have a cycle which is longer than $C$ ). Hence $G$ is

$$
\mathrm{K}_{\mathrm{k}} \mathrm{v} \mathrm{E}_{\mathrm{k}+1} \text {, where } \mathrm{n}=2 \mathrm{k}+1 \text {. }
$$

This completes the proof of Theorem 1.

QED

Proof of Theorem 2. Let G be a graph satisfying the conditions in Theorem 2. If G has a Hamiltonian path, then the proof is finished. Now we assume that $G$ is not traceable. Choose a longest path $P$ in $G$ and give an orientation on P. Let $y$ and $z$ be the two end vertices of $P$. We assume that the appearance of $y$ and $z$ on $P$ agrees with the given orientation of $P$. Since $G$ is not traceable, there exists a vertex $x_{0}$ in $V(G)-V(P)$. By Menger's theorem, we can find $s\left(s \geq k\right.$ ) pairwise disjoint (except for $x_{0}$ ) paths $P_{1}, P_{2}, \ldots, P_{s}$ between $x_{0}$ and $V(P)$. Let $u_{i}$ be the end vertex of $P_{i}$ on $P$, where $1 \leq i \leq s$. We, without loss of generality, assume that the appearance of $u_{1}, u_{2}, \ldots, u_{s}$ on $P$ agrees with the given orientation of $P$. Since $P$ is a longest path in $G, y \neq u_{i}$ and $z \neq u_{i}$, for each $i$ with $1 \leq i \leq s$, otherwise $G$ would have paths which are longer than $P$. We use $u_{i}{ }^{+}$to denote the successor of $u_{i}$ along the given orientation of $\mathrm{P}$, where $1 \leq \mathrm{i} \leq \mathrm{s}$. Then a standard proof in Hamiltonian graph theory yields that $\mathrm{S}:=\left\{\mathrm{x}_{0}, \mathrm{y}, \mathrm{u}_{1}^{+}, \mathrm{u}_{2}{ }^{+}\right.$, $\left.\ldots, \mathrm{u}_{\mathrm{k}}^{+}\right\}$is independent (otherwise $\mathrm{G}$ would have paths which are longer than P). Thus

$$
\begin{aligned}
& (n-k-2)(n+k+1) / 2 \leq e=|E(S, V-S)|+|E(G[V-S])| \\
& \leq|S|(n-|S|)+(n-|S|)(n-|S|-1) / 2 \\
& =(n-|S|)(n+|S|-1) / 2 \leq(n-k-2)(n+k+1) / 2 .
\end{aligned}
$$

Therefore $|S|=k+2$, xy in $E$ for any vertex $x$ in $S$ and for any vertex y in $V-S$, and $G[V-S)]$ is complete.

Let $\mathrm{H}$ be the component of $\mathrm{G}[\mathrm{V}-\mathrm{V}(\mathrm{P})]$ containing $\mathrm{x}_{0}$. Since $\mathrm{u}_{1}{ }^{+}$is adjacent to any vertex in $\mathrm{V}-\mathrm{S}, \mathrm{H}$ must consist of a singleton $\mathrm{x}_{0}$ (otherwise $G$ would have a path which is longer than P). Since $\mathrm{x}_{0}$ is adjacent to any vertex in $V-S$, $\mathrm{H}$ must be the only component of $\mathrm{G}[\mathrm{V}-\mathrm{V}(\mathrm{P})]$ (otherwise $\mathrm{x}_{0}$ would be adjacent to a vertex in another component of $G[V-V(P)]$, which is a contradiction). Again, since $x_{0}$ is adjacent to any vertex in $V-S$, the segment from $u_{i}^{+}$ to $u_{i+1}$ along the given orientation of $P$, for each i with $1 \leq i \leq s-1$, must consist of only $u_{i}^{+}$and $u_{i+1}$ (otherwise $G$ would have a path which is longer than P). Moreover, the segment from $y$ to $\mathrm{u}_{1}$ along the given orientation of $\mathrm{P}$ must consist of only y and $\mathrm{u}_{1}$ and the segment from $\mathrm{u}_{\mathrm{s}}{ }^{+}$to $\mathrm{z}$ along the given orientation of $\mathrm{P}$ must consist of only $\mathrm{u}_{\mathrm{s}}^{+}$. Hence $\mathrm{G}$ is

$$
\mathrm{K}_{\mathrm{k}} \mathrm{vE} \mathrm{k}_{2} \text {, where } \mathrm{n}=2 \mathrm{k}+2 \text {. }
$$

This completes the proof of Theorem 2.

QED

\section{REFERENCES}

1. J. A. Bondy and U. S. R. Murty, Graph Theory with Applications, Macmillan, London and Elsevier, New York (1976).

Citation: Rao Li, "New Sufficient Conditions for Hamiltonian and Traceable Graphs". American Research Journal of Mathematics; V3, I1; pp:1-2.

Copyright (c) 2017 Rao Li, This is an open access article distributed under the Creative Commons Attribution License, which permits unrestricted use, distribution, and reproduction in any medium, provided the original work is properly cited. 